\section{Orphaned by consumption - genomics of Mycobacterium tuberculosis and its relatives}

\author{
Genomics of GC-Rich Gram-Positive Bacteria \\ A Danchin \\ Caister Academic Press, New York. 2002; 178 pp. \\ $£ 75$, hardback. ISBN 0-9542464-3-8 \\ Reviewed by P Dyson
}

Heredity (2003) 90, 281. doi:10.1038/sj.hdy.6800230

To quote the blurb accompanying other titles in this series on Functional Genomics, 'genomics is a new and fast expanding area of biology encompassing high throughput or large-scale experimentation at the whole organism level, and the organisation, analysis and interpretation of the huge amount of data emerging from genome projects'. To date very few organisms have been investigated with these new approaches to the extent needed to give a more holistic view of their biology than previously possible. Perhaps more significant are results that suggest that the new genomic technologies often provide data sets that are inconsistent with each other, as can be the case, for example, when comparing transcriptome data based on microarrays derived from PCR products or long oligonucleotides. During the inevitable bedding-down period for these newly evolving technologies, it would be premature to expect sufficient output to warrant reviews that radically reassess our views of the biology of a given organism.

So potential readers of this volume should be prepared from the outset that this 'genomics of ...' account is a snapshot of the field taken at a very early, barely 'postgenomic' stage. Perhaps therein lies some confusion. 'Postgenomic' is a term used to describe investigations that follow on from reporting a fully sequenced and annotated genome, either based on predictions informed by the sequence or incorporating the sequence information in aspects of experimental design. In that much of the contents of this volume summarise the results of genome 'sequence-gazing', 'genomics of ...' may be an apt title. But the scope is limited largely to comparative genomics and does not address, for example, the 'orphans', genes whose function is unknown.

The GC-rich Gram-positive bacteria are actinomycetes, and examples of the phylum chosen for this volume are Rhodococcus, Mycobacterium, and Streptomyces. The choice of the former is somewhat eccentric: as yet there is no Rhodococcus genome sequence and even the traditional genetics of these interesting biodegrading bacteria is not well advanced. The Rhodococcus chapter is therefore distinctly 'pregenomic'. Other fully sequenced actinomycetes, such as Corynebacterium and Thermobifida, are overlooked.

Of the seven chapters in the book, four concern either Mycobacterium tuberculosis or its close relative $M$. leprae. Comparative genomics between the two indicates how the $M$. leprae genome has been paired down to the bare minimum that allows it still to grow, albeit at a very slow rate as an obligate intracellular pathogen. Comparison of the genomes of virulent and nonvirulent $M$. tuberculosis strains has been informative in identifying virulence determinants, in particular the ESAT-6 gene clusters. The reductive evolution that moulded the $M$. leprae genome should have been a lesson to the editor: a redundancy concerning the ESAT-6 genes is evident in three of the four mycobacterial chapters, with no cross-referencing between them. The repetition at least serves to emphasise the importance of these genes that may encode a novel type of macromolecular secretion system. Their functional analysis may, however, rely on investigations in the more tractable Streptomyces, a nonpathogenic model for Mycobacteria, perhaps better known for the production of the majority of antibiotics used in clinical and veterinary medicine. In terms of its size, the streptomycete genome is the 'mother' of actinomycete genomes, being almost twice that of $M$. tuberculosis and containing the largest number of orphans! The size is indicative of the enormous physiological and metabolic flexibility of these free-living complex soil bacteria. The architecture of the streptomycete genome is also noteworthy: the chromosome is atypically linear, with a conserved core of essential genes that has synteny with the chromosomes of other actinomycetes, and arms largely containing 'contingency' genes that are prone to deletions and amplifications. Incidentally, the reference for the Streptomyces genome sequence cited in the chapter on Rhodococcus is erroneous, referring as it does to the genome of Streptococcus pyogenes!

For those wishing to view the present snapshot, this book is reasonably informative. There is certainly scope for better editing and also more by way of comparative genomics between Mycobacteria and Streptomyces, if not other sequenced actinomycetes. In the meantime, we await the results of application of new functional genomic technologies to these fascinating bacteria with great interest.

P Dyson

School of Biological Sciences, University of Wales Swansea Singleton Park, Swansea SA2 8PP, UK E-mail: p.j.dyson@swansea.ac.uk 